\title{
ORIENTATION OF INTERCHANGE MULTIPLES IN SECALE CEREALE
}

\section{J. SYBENGA}

Department of Genetics, University of Agriculture, Wageningen, The Netherlands

Received 20.iv.67

\section{INTRODUGTION}

MEIotic pairing in interchange heterozygotes leads to the formation of multiple associations which at metaphase I may show two main types of orientation (fig. 1): alternate (disjunctional) and adjacent (non-disjunctional). In absence of crossing-over in the interstitial segment, alternate orientation results in balanced chromosome combinations, adjacent orientation results in unbalanced combinations. Thus the type of orientation affects

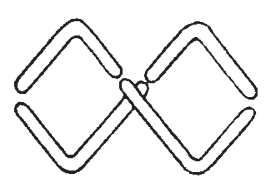

(a)

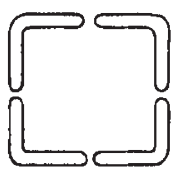

(b)

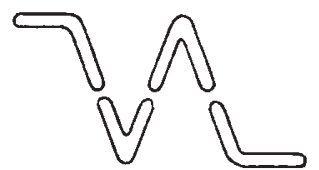

(c)

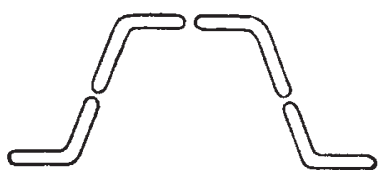

(d)

FIG. 1.-Four types of interchange orientations.
(a) Alternate ring.
(b) Adjacent ring.
(c) Alternate chain.
(d) Adjacent chain.

the genotype of the progeny and the fertility of the interchange heterozygote. In some organisms, adjacent and alternate orientations occur with about equal frequency which, in diploids with one interchange, results in approximately 50 per cent. fertility. In others the alternate type predominates, leading to less reduced or almost complete fertility. In such cases interchanges may be maintained in the population (Campanula, Gairdner and Darlington, 1931 ; Periplaneta, Lewis and John, 1957; Blaberus, John and Lewis, 1959), or even become fixed into the genetic system (Oenothera, Rhoeo). Such differences between species in respect of multivalent orientation are an indication of genetic determination, which has been demonstrated to exist within species (Secale cereale, Thompson, 1956; Lawrence, 1958, 1963). The reason why alternate orientation is more frequent in some genetic backgrounds than in others has been the subject of numerous speculations. Differences in chromosome rigidity, chiasma terminalisation or localisation, break-point or centromere location and the premetaphase stretch have been considered. In addition it has been observed that ring multivalents may have a different propensity for alternate orientation than chain multivalents. Lewis and John (1963) state that because of greater flexibility chain quadrivalents orientate alternately more frequently than ring quadrivalents. This agrees with the observation by Khoshoo and Mukherjee (1966) that in an interchange heterozygote of Canna, rings tend to orientate adjacently, chains orientating alternately. It contradicts the observations of Gairdner and 
Darlington (1931), who found a significantly $\left(\chi_{1}^{2}=10 \cdot 520\right)$ greater proportion of alternate segregation in rings than in chains of Campanula persicifolia. Müntzing and Prakken (1941) found the same tendency in rye interchanges, although their material was not large enough to show a significant difference $\left(\chi^{2}=2 \cdot 619\right)$. Lawrence $(1958,1963)$ could not demonstrate a difference between ring and chain quadrivalents in respect of orientation in rye, while in the present study on the same species rings were again found to show alternate orientation more frequently than chains. As shown below, these conflicting results may be reconciled.

\section{MAterials AND MEthods}

Two interchanges were studied: 248 (one plant) and 273 (three plants). One of these (248) involves the satellite of the satellite chromosome and the short arm of the chromosome with an arm length ratio of approximately 1.9 . The interchanged segments are relatively small and consequently the interstitial segments are relatively large. The satellite has gained a segment that is considerably larger than the segment it has lost. The other interchange (273) involves the long arm of the satellite chromosome and the short arm of the chromosome with an arm length ratio of approximately $1 \cdot 6$. Here the satellite chromosome has lost a larger segment than it has gained. The interchanged segments are somewhat larger than in the former case and the interstitial segments are smaller.

All plants were genetically heterozygous, although to varying extent, having resulted from intercrossing of different parental types. Those of 273 were somewhat related, the first two (273 I and 273 II) more closely than either one with the third (273 III and 273 IV). Only those cells were used in which the interchange complex was present as an open ring or an unbranched chain. These were by far the most frequent types. Cells with a trivalent, with only bivalents or with associations of four having chiasmata in interstitial segments, were not considered for the orientation studies.

In addition to the orientation types, the fraction of arms with at least one chiasma (bound arms, $b$ ) was determined both for the multiples and the non-interchange bivalents in cells that contained a multiple of any kind. For comparison, the frequencies of bound arms $(b)$ were transformed into crossing-over potentials $(\beta)$ ( $c f$. Sybenga, 1965).

The fixations of different plants were made at different times, those from the same plant were made simultaneously. In part, differences between plants may therefore be the result of environmental effects. Permanent aceto-carmine preparations after acetic alcohol (1:3) fixation were used. The same fixations of 273 have been reported upon earlier for other purposes (Sybenga, 1966).

\section{RESUlts}

The frequencies of the different orientation types for rings and chains are shown in the $2 \times 2$ contingency tables 1-6, together with the fraction of bound arms $(b)$ and the corresponding crossing-over potentials $(\beta)$. In respect of $b$ and $\beta$ it may be seen that the values for the interchange quadrivalents are lower than those for the non-interchange bivalents. This has earlier been ascribed to partner exchange interfering with chromosome pairing, resulting in lower chiasma frequencies (Sybenga, 1966). This 
reduction is similar in $273 \mathrm{I}$ and $273 \mathrm{II}$, two closely related plants, and somewhat different in 273 III, a more distantly related plant. The observations on 248 (one plant) are again reasonably close together. With progressing metaphase the number of bound arms, and consequently the estimates of the crossing-over potentials, decrease in a similar way in bivalents and multiples of four (tables 1, 2, 5 and 6). It may be ascribed to chiasmata slipping off the chromosome ends (Sybenga, 1966), leading to an increase of the number of chains at the expense of the rings.

TABLE 1

Multiple (IV) orientation at early metaphase in heterozygote 248. Probabilities of being bound (b) and crossing-over potentials $(\beta)$ for multivalent and non-interchange bivalent arms. Cells without multiples or with multiples having interstitial chiasmata are excluded

Orientation

Rings

Chains

Total

Alternate

Adjacent

$143(76 \cdot 1 \%)$

$119(52 \cdot 9 \%)$

106

$262(63 \cdot 4 \%)$

$\chi_{1}^{2}=23 \cdot 716$

Total

188

225

413

$b_{\text {biv. }}=0.951 ; b_{\text {mult. }}=0.853 ; \beta_{\text {biv. }}=1.508 ; \beta_{\text {mult. }}=0.959 ; \frac{\beta_{\text {biv. }}}{\beta_{\text {mult. }}}=1.57$.

TABLE 2

Multiple (IV) orientation at late metaphase in heterozygote 248. For further legend see table 1. Another anther from same floret as that of table 1

Orientation

Alternate

Adjacent

Total
Rings

$155(93 \cdot 4 \%)$

11

166
Chains

$193(79 \cdot 8 \%)$

49

242
Total

$348(85 \cdot 3 \%) \quad \chi_{1}^{2}=14 \cdot 565$
60

408

$$
b_{\text {biv. }}=0.934 ; b_{\text {mult. }}=0.842 ; \beta_{\text {biv. }}=1.359 ; \beta_{\text {mult. }}=0.923 ; \frac{\beta_{\text {blv. }}}{\beta_{\text {mult. }}}=1.47 .
$$

TABLE 3

Multiple (IV) orientation in heterozygote 273, plant I. For further legend see table 1

Orientation

Alternate

Adjacent

Total
Chains

$184(87 \cdot 6 \%)$

26

210
Total

$898(91 \cdot 4 \%) \quad \chi_{1}^{2}=4 \cdot 713$

85

983

$$
b_{\text {biv. }}=0.989 ; b_{\text {mult. }}=0.941 ; \beta_{\text {biv. }}=2.255 ; \beta_{\text {mult. }}=1.415 ; \frac{\beta_{\text {biv. }}}{\beta_{\text {mult. }}}=1.59 \text {. }
$$

In respect of orientation three main conclusions are evident:

1. Alternate orientation predominates, in the present material varying between 53 and 95 per cent.

2. Alternate orientation is more frequent in rings than in chains except in one case (table 2), a late metaphase.

3. Alternate orientation is more frequent in later than in earlier stages of M I. 
In connection with conclusions 2 and 3 it is of interest to see whether rings or chains contribute most to the increase in alternate orientation with preceding M I. This may be done by comparing early and late metaphase in 248 (tables 1 and 2) and in 273 III (tables 5 and 6). In both cases the change appears to be more pronounced in chains than in rings, especially

TABLE 4

Multiple (IV) orientation in heterozygote 273 , plant II. For further legend see table 1

Orientation

Rings

Alternate

Adjacent

Total
Chains

$267(87 \cdot 0 \%)$

307
Total

$914(92 \cdot 4 \%) \quad \chi_{1}^{2}=18 \cdot 840$

989

$b_{\text {biv. }}=0.979 ; b_{\text {mult. }}=0.920 ; \beta_{\text {biv. }}=1.941 ; \beta_{\text {mult. }}=1.264 ; \frac{\beta_{\text {biv. }}}{\beta_{\text {mult. }}}=1.54$.

\section{TABLE 5}

Multiple (IV) orientation at early metaphase in heterozygote 273, plant III. For further legend see table 1

\begin{tabular}{llccr} 
Orientation & \multicolumn{1}{c}{ Rings } & Chains & Total \\
Alternate & $727(86 \cdot 1 \%)$ & $100(69.9 \%)$ & $827(83.8 \%)$ & $\chi_{\mathbf{1}}^{2}=23.648$ \\
Adjacent & 117 & 43 & 160 \\
otal & 844 & 143 & 987 \\
$\quad b_{\text {biv. }}=0.971 ;$ & $b_{\text {mult. }}=0.961 ;$ & $\beta_{\text {biv. }}=1.770 ;$ & $\beta_{\text {mult. }}=1.625 ;$ & $\beta_{\text {biv. }}=1.09$.
\end{tabular}

TABLE 6

Multiple (IV) orientation at late metaphase in heterozygote 273 , plant III. For further legend se table 1. Other anther from same inflorescence as that of table 5

\section{Orientation}

Alternate

Adjacent

Total

$b_{\text {biv. }}=0.944 ; b_{\text {mult. }}=0 \cdot 919 ;$
Chains

311
28

339

$141(91 \cdot 6 \%)$
13

154
Total

$$
\begin{aligned}
& 452(91 \cdot 7 \%) \quad \chi_{1}^{2}=0.003 \\
& 41 \\
& 493
\end{aligned}
$$

in 273. In tables 1 and 2 the effect is somewhat more obscure, the difference between " early" and "late" being smaller, but even after angular transformation of the percentages of alternate orientation the difference is greater for chains than for rings. Tests indicate a very low probability indeed of the effect being due to chance alone (tables 7 and 8 ).

\section{Discussion}

The present results confirm the earlier observations (Müntzing and Prakken, 1941; Thompson, 1956; Lawrence, 1958, 1963) that the orientation of interchange multiples in rye is predominantly alternate. They contradict the suggestion made by Lewis and John (1963) that chain 


\section{TABle 7}

Calculation of $\chi^{2}$ for the difference between ring and chain multiples in their change from adjacent to alternate orientation with progressing metaphase I. Heterozygote 248

\begin{tabular}{lcccccc}
\multicolumn{1}{c}{ M I } & $\begin{array}{c}\text { Rings } \\
\text { alternate }\end{array}$ & $\begin{array}{c}\text { Rings } \\
\text { adjacent }\end{array}$ & $\begin{array}{c}\text { Chains } \\
\text { alternate }\end{array}$ & $\begin{array}{c}\text { Chains } \\
\text { adjacent }\end{array}$ & Total & \\
Early & 143 & 45 & 119 & 116 & 413 & $\chi_{1}^{2}=59 \cdot 610$ \\
Late & 155 & 11 & 193 & 49 & 408 & \\
Total & 298 & 56 & 312 & 155 & 821 &
\end{tabular}

M I Alternate Adjacent Total

$\begin{array}{lrrrr}\text { Early } & 262 & 151 & 413 & \chi_{1}^{2}=51 \cdot 342 \\ \text { Late } & 348 & 60 & 408 & \end{array}$

$\begin{array}{lll}\text { Total } & 610 & 211\end{array}$

M I Rings Chains Total

$\begin{array}{lllll}\text { Early } & 188 & 225 & 413 & \chi_{1}^{2}=1.956 \\ \text { Late } & 166 & 242 & 408 & \end{array}$

$\begin{array}{llll}\text { Total } & 354 & 467 & 821\end{array}$

Interaction $\chi_{1}^{2}=6 \cdot 312$

TABLE 8

Calculation of $\chi^{2}$ for the difference between rings and chains of four in their change from adjacent to alternate orientation with progressing metaphase I. Interchange 273, plant III

\begin{tabular}{|c|c|c|c|c|c|}
\hline M I & $\begin{array}{l}\text { Rings } \\
\text { alternate }\end{array}$ & $\begin{array}{l}\text { Rings } \\
\text { adjacent }\end{array}$ & $\begin{array}{l}\text { Chains } \\
\text { alterante }\end{array}$ & $\begin{array}{c}\text { Chains } \\
\text { adjacent }\end{array}$ & Total \\
\hline Early & 727 & 117 & 100 & 43 & \multirow{2}{*}{$\chi_{1}^{2}=89.474$} \\
\hline Late & 311 & 28 & 141 & 13 & \\
\hline \multirow[t]{10}{*}{ Total } & 1038 & 145 & 241 & 56 & 1480 \\
\hline & M I & Alternate & Adjacent & Total & \multirow{4}{*}{$\chi_{1}^{2}=17 \cdot 458$} \\
\hline & Early & 827 & 160 & 987 & \\
\hline & Late & 452 & 41 & 493 & \\
\hline & Total & 1279 & 201 & 1480 & \\
\hline & M I & Rings & Chains & Total & \multirow{4}{*}{$\chi_{1}^{2}=57 \cdot 499$} \\
\hline & Early & 844 & 143 & 987 & \\
\hline & Late & 339 & 154 & 493 & \\
\hline & Total & 1183 & 297 & 1480 & \\
\hline & & & & Interact & $x_{1}^{2}=14.517$ \\
\hline
\end{tabular}


quadrivalents generally orientate alternately more frequently than do ring quadrivalents. On the whole they also seem to contradict the conclusion of Lawrence (1963) that in rye chain multiples orientate alternately as frequently as do rings, but in this respect confirm the observations of Müntzing and Prakken (1941). There is one exception (table 2) where chains and rings have the same, high, percentage of alternate orientation. In this case the cells were approaching anaphase. As chains of four tend to continue to change their orientation to a greater degree during the course of M I than do rings, it is possible that the material studied by Lawrence (1963) consisted mainly of rather late cells. For fertility and segregation only the final orientation is significant. Of course, genetic and environmental differences may also play a part. Although there are some indications of genetic effects, the present material was not suitable for an analysis.

Neither interchange conformed entirely to the requirements formulated for instance by Burnham (1956) for high frequency of alternate orientation. Chiasma terminalisation is usually, but not always, rather complete. Premetaphase stretch does not occur to an appreciable extent, which Lewis and John (1957) and John and Lewis (1959) consider to be of special importance for the predominantly alternate orientation in Periplaneta and Blaberus.

The present results are perhaps best explained by the special opportunity rye centromeres appear to have of changing their orientation from one pole to the other even during metaphase, in addition to some rigidity of the chromosome at this stage, except at the centromeric region. It could be visualised that a change of orientation of individual centromeres is realised more easily in an adjacent orientation than in an alternate one. A centromere that changes its orientation from one pole to another in an alternate ring (fig. l, a) always has two arms that oppose this movement, the direction of their axes being near to that of the movement of the centromere. In an adjacent ring there is only one such arm for each centromere, the other being no obstacle to reorientation (fig $1, \mathrm{~b}$ ). Thus there will be a strong tendency for alternate orientation to be maintained, while adjacent orientation may change more easily. Alternate chain quadrivalents (fig. 1, c) are less rigid and may not be as easily maintained as rings, but still offer more resistance to reorientation than adjacent chains (fig. 1, d). Therefore chains take longer to reach the (same?) final level of alternate orientation at an equilibrium situation.

The slipping of all chiasmata off the ends of a paired arm transforms a ring into a chain. If this process is enhanced by the stress exerted on the arms by the centromeric forces, adjacent rings are expected to loose their chiasmata more easily than alternate rings, where four instead of two arms are available to resist the stress. The resulting adjacent chains may have better opportunities to change their orientation than had the original rings. This may contribute to the general change towards alternate orientation observed for rings and the lower level of alternate orientation of chains. On the other hand, it has been noted that adjacent chains practically never lack chiasmata between the co-oriented centromeres (fig. l, d), nor has linear orientation been observed as would be expected when adjacent rings lose their chiasmata as a result of centromeric forces. Therefore either only alternate rings change into chains or newly arisen adjacent chains immediately reorientate their centromeres. The latter may be the more plausible explanation. 
Genetic variation in chromosome rigidity and in the capacity of the centromeres to reorientate must be the cause of the observed genetically conditioned variations in preference for a certain orientation type. A relation between the system operating here and premetaphase stretch (Lewis and John, 1957) is not obvious. Perhaps the observation that reorientation of centromeres after premetaphase stretch is possible, points to some similarity. The case of Canna (Khoshoo and Mukherjee, 1966), where rings of four generally had an adjacent orientation and chains an alternate orientation, requires the assumption that with these small and contracted chromosomes, arms and centromeric regions are too rigid to permit an easy realisation of alternate orientation in a ring, while the rigidity of the chains is sufficiently less. The rigidity of chains, however, may here be sufficient to maintain the alternate orientation once it has come about in spite of tendencies of the centromeres to reorientate.

\section{Summary}

1. Multiples of two different interchanges in rye showed preferential alternate orientation (up to 95 per cent.) although they did not show the features commonly believed to favour this behaviour.

2. Alternate orientation was more common in rings than chains but the difference was less marked at late metaphase owing to the more frequent reorientation of chains from adjacent to alternate.

3. Alternate orientations offer more resistance to reorientation than adjacent orientations, more so in rings than in chains. Therefore at equilibrium alternate orientations will predominate both for rings and for chains, but chains reach equilibrium later than rings.

\section{ReFERENGES}

BURnham, c. R. 1956. Chromosomal interchanges in plants. Bot. Rev., 22, 419-552.

GAIRDNER, A. E., AND DARLINGTON, C. D. 1931. Ring formation in diploid and polyploid Campanula persicifolia. Genetica, 13, 113-150.

JOHN, B., AND LEWIS, K. R. 1959. Selection for interchange heterozygosity in an inbred culture of Blaberus discoidalis (Serville). Genetics, 44, 251-267.

Khoshoo, т. N., AND mUKherjee, I. 1966. A translocation heterozygote in garden Canna. Genetica, 37, 255-258.

LAWRENCE, c. W. 1958. Genotypic control of chromosome behaviour in rye. VI. Selection for disjunction frequency. Heredity, 12, 127-131.

LAWRENCE, c. W. 1963. The orientation of multiple associations resulting from interchange heterozygosity. Genetics, 48, 347-350.

LEWIS, K. R., AND JOHN, B. 1957. Studies in Periplaneta americana. II. Interchange heterozygosity in isolated populations. Heredity, 11, 11-21.

LEWIS, K. R., AND JOHN, B. 1963. Chromosome Marker. J. \& A. Churchill Ltd., London. MÜNTZING, A., AND PRAKKEN, R. 1941. Chromosomal aberrations in rye populations. Hereditas, 27, 273-308.

SYBENGA, J. 1965. The quantitative analysis of chromosome pairing and chiasma formation based on the relative frequencies of M I configurations. I. Introduction: normal diploids. Genetica, 36, 243-252.

SYBENGA, J. 1966. The quantitative analysis of chromosome pairing and chiasma formation based on the relative frequencies of $\mathbf{M} I$ configurations. V. Interchange trisomics. Genetica, 37, 481-510.

тHOMPSON, J. B. 1956. Genotypic control of chromosome behaviour in rye. II. Disjunction at meiosis in interchange heterozygotes. Heredity, 10, 99-108. 